\title{
Duopoly Market Analysis within One-Shot Decision Framework with Asymmetric Possibilistic Information
}

\author{
Peijun Guo \\ Faculty of Business Administration, \\ Yokohama National University \\ 79-4 Tokiwadai, Hodogaya-ku, Yokohama, 240-8501 Japan \\ E-mail: guo@ynu.ac.jp \\ Ruiliang Yan \\ School of Business and Economics \\ Indiana University Northwest \\ Gary, IN 46408, USA \\ E-mail: ruiliangy@gmail.com \\ John Wang \\ Department of Management and Information Systems \\ Montclair State University \\ Montclair, NJ 07043 USA \\ j.john.wang@gmail.com \\ Received: 24-02-2010 \\ Accepted: 05-10-2010
}

\begin{abstract}
In this paper, a newly emerging duopoly market with a short life cycle is analyzed. The partially known information of market is characterized by the possibility distribution of the parameter in the demand function. Since the life cycle of the new product is short, how many products should be produced by two rival firms is a typical one-shot decision problem. Within the one-shot decision framework, the possibilistic Cournot equilibrium is obtained for the optimal production level of each firm in a duopoly market with asymmetrical possibilistic information. The analysis results show that the proposed approaches are reasonable for one-shot decision problems, which are extensively encountered in business and economics.
\end{abstract}

Keywords: Duopoly market, focus points, one-shot decision, possibilistic Cournot equilibrium, possibility theory.

\section{Introduction}

Decision analysis is choosing among alternatives considering information about the states of nature, which are the factors beyond the control of a decision maker. As a limiting case, a decision maker may know which state of nature will occur. In such a case (known as decision making under certainty), it is easy to make a choice because the outcome of each alternative is deterministic. Otherwise, a decision maker acts under non-certainty. Non-certainty is divided into three categories -risk, uncertainty, and ignorance. Situations involving risk are when the probabilities of all possible outcomes can be exactly calculated. On the other hand, uncertain situations are associated with partially known information when probabilities cannot be obtained exactly [21]. Situations involving ignorance occur when no information is available to distinguish which state of nature is more likely to occur.

Decision rules for situations involving ignorance include maximin, maximax, minmax regret and Hurwicz criterion. Leading theories of decision include 
the expected utility (EU) theory of von Neumann and Morgenstern and the subjective expected utility (SEU) theory of Savage. EU is used in decision-making under risk whereas SEU deals with decision-making under uncertainty. Subjective probabilities are used to reflect a decision-maker's belief, traditionally analyzed in terms of betting behavior. The SEU axioms show the conditions under which preferences can be represented by a numerical expected utility that uses subjective probabilities of the states of nature to weight consequence utilities.

Research has shown that SEU does not provide an adequate description of individual choice under uncertainty $[1,5]$. Alternative models such as regret theory [2, 23], second-order probabilities [15, 26], and non-additive probability models $[6,25]$ have been proposed in this empirical challenge. Prospect theory developed by Kahneman and Tversky [16] is a noble non-additive probability model. Under prospect theory, value is assigned to gains and losses based on reference point rather than to final assets as in EU and SEU. Also probabilities are replaced by decision weights without additivity. Value functions are normally concave for gains (implying risk aversion), and convex for losses (risk seeking)

Possibility theory, initiated by Zadeh [30] and advanced by Dubois, Prade and Klir [3, 20] is one of the current uncertainty theories devoted to handling of incomplete information in the real world. Possibility theory is based on two basic non-additive measures, possibility measure and necessity measure. Decision analysis under uncertainty also can be found in the literatures [7-8, 17-19, 24, 27-29].

It is known that decision theories under uncertainty are theories of choice under uncertainty where the objects of choice are probability distributions (for EU, SEU and their varieties), prospects framed in terms of gains and losses (for prospect theory), or possibility distributions (regarded as possibilistic lotteries [4]). In fact, for a one-shot decision problem which is for the situation that decision is experienced once only, there is one and only one chance for one state of nature coming up. Guo [9-11] initially proposed the one-shot decision approaches for such decision problems. The procedure for one-shot decision is separated into two steps. The first step involves focusing some states of nature, called focus points. Which state of nature is focused characterizes a decision maker's different attitudes about satisfaction and possibility. In the second step, alternatives are evaluated based on their focus points where the satisfaction levels provided by all alternatives are compared with each other to obtain the optimal alternative.

Within the one-shot decision framework, a duopoly market of a new product with a short life cycle is analyzed. The possibilistic Cournot equilibrium is proposed to analyze the optimal production level of each firm in a duopoly market [9]. This paper is its extension where the procedure for calculating the possibilistic Cournot equilibrium is proposed and the extension of possibilistic Cournot equilibrium with asymmetrical possibilistic information is made.

This paper is organized as follows: Section 2 introduces one-shot decision framework. In Section 3, possibilistic decision models for a duopoly market are addressed where a duopoly market with asymmetrical possibilistic information is investigated. Finally, concluding remarks for this research are made in Section 4.

\section{One-Shot Decision Framework}

\subsection{Possibility distribution and Satisfaction Function}

In one-shot decision problems, the set of an alternative $a$ is $A$. The set of a state of nature $x$ is $S$. The degree to which a state of nature is to occur in the future is characterized by a possibility distribution $\pi(x)$, as defined below.

Definition 1. Given a function

$$
\text { if } \begin{gathered}
\pi: S \rightarrow[0,1] \\
\max _{x \in S} \pi(x)=1,
\end{gathered}
$$

then the function $\pi(x)$ is called a possibility distribution where $S=\{x\}$ is the sample space. $\pi(x)$ is the possibility degree of $x . \pi(x)=1$ means that it is normal that $x$ occurs and $\pi(x)=0$ means that it is abnormal that $x$ occurs. The smaller the possibility degree of $x$, the more surprised for the happening of $x$. The following example shows how to estimate the uncertain demand by the possibility distribution.

Example 1. The demand estimation of a fashion clothes. We ask 160 young female customers whether they are satisfied with a particular type of fashion clothes. The 
items of questionnaire include categories such as price, style, color and comfort. The total satisfaction degree of a customer is determined by the minimum of his satisfaction levels of all categories. The numbers of the customers whose satisfaction levels are $1,0.8,0.7,0.6$, 0.5 and the other are 50, 40, 30, 20, 10 and 10, respectively. Based on such results, an experienced salesman is asked the following questions about his level of surprise.

Q1: How surprised are you if only the customers with full satisfaction levels (1.0) buy the clothes?

A1: Very surprised (far different from the experience of this salesman).

Q2: How surprised are you if only the customers with satisfaction levels not less than 0.8 buy the clothes?

A2: A litter bit surprised (different from the experience of this salesman).

Q3: How surprised are you if only the customers with satisfaction levels not less than 0.7 buy the clothes?

A3: Not surprised (matches the experience of this salesman).

Q4: How surprised are you if all the customers with satisfaction levels not less than 0.6 buy the clothes?

A4: A litter bit surprised.

Q5: How surprised are you if all the customers with satisfaction levels not less than 0.5 buy the clothes?

A5: Very surprised.

Denote the degree of surprise as $t$. It is the common sense that the more surprised, the more impossible. Thus it is reasonable to define the possibility degree as $1-t$. If we set $t$ as $1,0.25,0$ for the situations of "very surprised", "a litter bit surprised", "not surprised", respectively, then the possibility degrees of demands of $50,90(=50+40), 120(=50+40+30), 140(=50+40+30+20)$, $150(=50+40+30+20+10)$ are $0,0.75,1,0.75,0$, respectively.

From the above example, it is clear that the possibility distribution can be used to represent the knowledge or judgment of human being. In the literature [12], the upper and lower exponential possibility distributions are obtained by possibilistic linear programming problems to reflect the feasible region of decision variables. In the literatures [13-14], the upper and lower exponential possibility distributions are identified from the given possibility degrees of samples by experts via linear programming problems and the identified possibility distributions of the securities' returns are used for portfolio selection problems.

The consequence resulting from the combination of an alternative $a$ and a state of nature $x$ is refereed to as a payoff, denoted as $v(x, a)$. The satisfaction level of a decision maker for a payoff can be expressed by a satisfaction function, as defined below.

Definition 2. Denote the set of a payoff $v(x, a)$ as $V$. The following function

$$
u: V \rightarrow[0,1]
$$

with

$$
u\left(v_{1}\right)>u\left(v_{2}\right) \text { for } v_{1}>v_{2},
$$

is called a satisfaction function. Because the payoff is the function of $x$ and $a$, we can rewrite the satisfaction function as $u(v(x, a))$. For the sake of simplification, sometimes we write $u(v(x, a))$ as $u(x, a)$ in this paper.

The information for one-shot decision can be summarized as a quadruple $(A, S, \pi, u)$. One-shot decision is to choose one alternative based on $(A, S, \pi, u)$ when only one decision chance is given. Since one and only one state of nature will occur for one-shot decision, the decision maker should first consider which state of nature should be focused for making a decision. The focused states of nature are called focus points. The procedure for choosing focus points is introduced in the following subsection.

\subsection{Determining Focus Points of Alternatives}

Let us begin with the following definition which is used for determining focus points.

Definition 3. Given the vector $\left[a_{1}, a_{2}, \cdots, a_{n}\right]$, $\min \left[a_{1}, a_{2}, \cdots, a_{n}\right] \quad$ and $\max \left[a_{1}, a_{2}, \cdots, a_{n}\right]$ are defined as follows:

$$
\begin{aligned}
& \min \left[a_{1}, a_{2}, \cdots, a_{n}\right]=\left[\underset{i=1, \ldots, n}{\wedge} a_{i}, \wedge a_{i=1, \ldots, n}, \cdots, \underset{i=1, \ldots, n}{\wedge} a_{i}\right], \\
& \max \left[a_{1}, a_{2}, \cdots, a_{n}\right]=\left[\underset{i=1, \ldots, n}{\vee} a_{i=1, \ldots, n}, \vee a_{i}, \cdots, \underset{i=1, \ldots, n}{\vee} a_{i}\right] .
\end{aligned}
$$

$\min \left[a_{1}, a_{2}, \cdots, a_{n}\right]$ and $\max \left[a_{1}, a_{2}, \cdots, a_{n}\right]$ are the lower and upper bounds of $\left[a_{1}, a_{2}, \cdots, a_{n}\right]$, respectively. For example, $\min [0.3,0.8]=[0.3,0.3] \quad$ and $\max [0.3,0.8]=[0.8,0.8]$.

Choice 1. A decision maker only focuses on the normal case so that the state of nature with the possibility degree 1 , denoted as $x^{\circ}$, is taken into account which is

$$
x^{o}=\arg \max _{x \in S} \pi(x) .
$$


$x^{o}$ is called a normal focus point. $\arg \max _{x \in S} \pi(x)$ is used to denote the value of $x$ which make $\pi(x)$ maximize.

Choice 2. For an alternative $a$, a decision maker focuses a state of nature, denoted as $x^{*}(a)$, as follows:

$$
x^{*}(a)=\arg \max _{x \in S} \min [\pi(x), u(x, a)] \text {. }
$$

It follows from (8) that $x=x^{*}(a)$ maximizes $g(x, a)=\min [\pi(x), u(x, a)]$. In consideration of (5), we know that $\min [\pi(x), u(x, a)]$ represents the lower bound of the vector $[\pi(x), u(x, a)]$; that is, for a state of nature $x$, both of the possibility degree and the satisfaction level provided by an alternative $a$ are at least $\quad \min (\pi(x), u(x, a)) \quad$. $\quad$ Increasing $\min [\pi(x), u(x, a)] \quad\left(\max _{x \in S} \min [\pi(x), u(x, a)]\right) \quad$ will increase the lower bounds of the possibility degree and the satisfaction level simultaneously. Therefore, $\arg \max _{x \in S} \min [\pi(x), u(x, a)]$ is for seeking the state of nature that has the higher possibility degree and the higher satisfaction level. The obtained state of nature $x^{*}(a)$ is called an active focus point of an alternative $a$.

Choice 3. For an alternative $a$, a decision maker focuses a state of nature, denoted as $x_{*}(a)$, as follows:

$$
x_{*}(a)=\arg \min _{x \in S} \max [1-\pi(x), u(x, a)] \text {. }
$$

It follows from (9) that $x=x_{*}(a)$ minimizes $k(x, a)=\max [1-\pi(x), u(x, a)] \quad . \quad$ Since $\max [1-\pi(x), u(x, a)]$ represents the upper bound of the vector $[1-\pi(x), u(x, a)] \quad, \quad$ decreasing $\max [1-\pi(x), u(x, a)] \quad\left(\min _{x \in S} \max [1-\pi(x), u(x, a)]\right)$ will decrease the upper bounds of $1-\pi(x)$ and $u(x, a)$ simultaneously. In other words, $\min _{x \in S} \max [1-\pi(x), u(x, a)]$ will increase the lower bound of the possibility degree $\pi(x)$ and decrease the upper bound of the satisfaction level $u(x, a)$. Therefore, $\arg \min _{x \in S} \max [1-\pi(x), u(x, a)]$ is for seeking the state of nature that has the higher possibility degree and the lower satisfaction level. The obtained state of nature $x_{*}(a)$ is called a passive focus point of an alternative $a$.

\subsection{Obtaining the Optimal Alternative}

Based on the obtained different types of focus points, the optimal alternatives are determined as follows:

$$
\begin{aligned}
& a^{o}=\arg \max _{a \in A} \min _{x^{o} \in X^{o}} u\left(x^{o}, a\right), \\
& a^{*}=\arg \max _{a \in A} \max _{x^{*}(a) \in X^{*}(a)} u\left(x^{*}(a), a\right), \\
& a_{*}=\arg \max _{a \in A} \min _{x_{*}(a) \in X^{*}(a)} u\left(x_{*}(a), a\right),
\end{aligned}
$$

where $X^{o}$ is the set of normal focus points, $X^{*}(a)$ and $X_{*}(a)$ are the sets of active and passive focus points of an alternative $a$, respectively. $a^{o}, a^{*}$ and $a_{*}$ are called normal, active and passive optimal alternatives, respectively. $x^{*}\left(a^{*}\right)$ and $x_{*}\left(a_{*}\right)$ are called optimal active and optimal passive focus points, respectively. Decision rules maximin and maximax are used in (10), (11) and (12) for the cases that multiple focus points exist for an alternative. maximin and maximax reflect the conservative and aggressive attitude, respectively.

A decision maker is called a normal decision maker or an active decision maker or a passive decision maker if he takes into account the normal focus points or active focus points or passive focus points for making one-shot decisions. We can image three types of decision makers as follows: when making a one-shot decision the normal decision maker focuses on the most possible outcome; the active decision maker takes into account the scenario which can yield the higher satisfaction with a higher possibility; the passive decision maker considers the scenario which can lead to the lower satisfaction with a higher possibility.

\section{Theorem 1 [9].}

\section{Assume that}

(I) Possibility distribution is a unimodal continuous function which satisfies $\pi\left(x_{l}\right)=0, \pi\left(x_{u}\right)=0$ and $\pi\left(x_{c}\right)=1$ and $\pi(x)$ increases within $\left[x_{l}, x_{c}\right]$ and decreases within $\left[x_{c}, x_{u}\right]$, where $x_{c}, x_{l}$ and $x_{u}$ are the center, lower and upper bounds of $x$.

(II) $u(x, a)$ and $\max _{a \in A} u(x, a)$ are continuous, strictly increasing, functions of $x$, respectively.

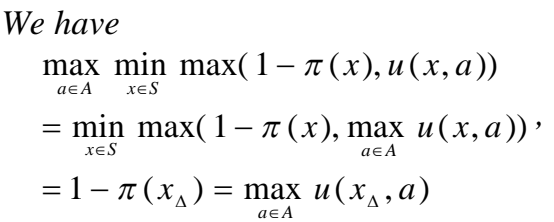




$$
\begin{aligned}
& \max _{a \in A} \max _{x \in S} \min (\pi(x), u(x, a))=\pi\left(x^{\Delta}\right), \\
& =\max _{a \in A} u\left(x^{\Delta}, a\right)
\end{aligned}
$$

where $x_{\Delta}$ is the horizontal coordinate of the unique intersection of $1-\pi(x)$ and $\max _{a \in A} u(x, a)$ within $\left[x_{l}, x_{c}\right]$, and $x^{\Delta}$ is the horizontal coordinate of the unique intersection of $\pi(x)$ and $\max _{a \in A} u(x, a)$ within $\left[x_{c}, x_{u}\right]$.

\section{Duopoly Market Analysis with Possibilistic Information}

\subsection{The Basic of Cournot Equilibrium}

We image an industry with two firms, 1 and 2 (duopoly market), each producing and selling single goods. Consumers do not care from which firm they purchase the goods. The following well-used linear inverse demand function in economics is considered [22]:

$$
p=c-d\left(q_{1}+q_{2}\right),
$$

where $p>0$ is a price, $q_{1}$ and $q_{2}$ are production levels of Firm 1 and Firm 2, respectively. $c>0$ is the p-interept of (15) representing the limit price of goods when the total supply approaches to zero and $d>0$ is the slope of (15) showing the price decrease when the total supply increasing by one unit.

Here we consider that each firm has Cournot conjecture about its rival: Each of two firms assumes that the other firm will act in a way to keep fixed the production level. With Cournot conjecture, the profit of each firm $i(i=1,2)$ is as follows:

$$
w_{i}\left(c, q_{i}, q_{j}\right)=c q_{i}-d q_{i}^{2}-d q_{i} q_{j}, i \neq j
$$

where the production cost is ignored for simplicity.

Conjecturing that Firm $j$ has the production level $q_{j}$, the sufficient and necessary condition for maximizing (16) is as follows:

$$
d w_{i} / d q_{i}=c-2 d q_{i}-d q_{j}=0, i \neq j,
$$

which is obtained by differentiating (16) with $q_{i}$. So that the optimal production level of Firm $i$ with the parameters $q_{j}$ and $c$, denoted as $q_{i}^{\nabla}\left(q_{j}, c\right)$ is obtained as

$$
q_{i}^{\nabla}\left(q_{j}, c\right)=\left(c-d q_{j}\right) / 2 d, i \neq j .
$$

Two firms are at equilibrium in this industry when neither firm wants to change what it is doing, given that the rival keeps the same production level. So that this equilibrium $\left(q_{1}^{\Delta}, q_{2}^{\Delta}\right)$, called Cournot equilibrium, is the solution of the following equations

$$
\left\{\begin{array}{l}
q_{1}=\left(c-d q_{2}\right) / 2 d \\
q_{2}=\left(c-d q_{1}\right) / 2 d
\end{array},\right.
$$

which leads to

$$
q_{1}^{\Delta}=q_{2}^{\Delta}=\frac{c}{3 d}
$$

where $q_{i}^{\Delta}$ is called the equilibrium production level of Firm $i$.

\subsection{Cournot Equilibrium with Possibilistic Information}

Let us consider a newly emerging duopoly market with a short life cycle. It is reasonable to think that there is only one chance for these two firms to decide how many products they should produce due to a short life cycle. Because the product is new, no relevant historical data can be used for statistical analysis in this case. However, marketing research can obtain some information about what is the most possible situation and what is impossible one in the future. Such plausible information can be characterized by the possibility distribution of $c$ as follows:

The possibility distribution of $c$ is set as the following continuous unimodal function

$$
\pi:\left[c_{l}, c_{r}\right] \rightarrow[0,1],
$$

where $c_{l}$ and $c_{r}$ are the lower and upper bounds of $c$. $\exists c_{o} \in\left[c_{l}, c_{u}\right]$ so that $\pi\left(c_{o}\right)=1, \pi\left(c_{l}\right)=0$ and $\pi\left(c_{r}\right)=0 . \pi(c)$ increases within $\left[c_{l}, c_{o}\right]$ and decreases within $\left[c_{o}, c_{r}\right]$.

Remark 1: Considering (16) and (18), the maximal profit of Firm $i$ with $q_{j}$ is as follows:

$$
\max _{q_{i}} w_{i}\left(c, q_{i}, q_{j}\right)=\frac{\left(c-d q_{j}\right)^{2}}{4 d} .
$$

(22) shows that the maximum profit of Firm $i$ for a given $c$ is $\frac{c^{2}}{4 d}$. The profit of Firm $i$ reaches its minimum 0 when $q_{i}=0$. Considering the region of $c$, the region of the profit of Firm $i$ is $\left[0, \frac{c_{r}^{2}}{4 d}\right]$. It is easy to know that the region of the production levels of two firms is $\left[0, \frac{c_{r}}{d}\right]$. 
The satisfaction function of Firm $i$, denoted as $u\left(w_{i}\left(q_{i}, q_{j}, c\right)\right)$, is a strictly increasing function of $w_{i}$ with the conditions $u(0)=0$ and $u\left(\frac{c_{r}^{2}}{4 d}\right)=1$. Let us analyze the duopoly market problem within the one-shot decision framework. Two firms have ability to produce a new product with a short life cycle. The types of firms are normal, active and passive. Such a type is regarded as an inherent feature of a firm. A firm knows his own type but does not know the type of its rival. The information $(A, S, \pi, u)$ is common knowledge for both of firms where $A=\left[0, c_{r} / d\right], S=\left[c_{l}, c_{r}\right]$. Based on $(A, S, \pi, u)$ and his own type, the firm should make a one-shot decision on the production level to maximize its profit with Cournot conjecture about its rival.

From Choice 1 we know that the normal focus point is $c_{o}$. Considering Choice 2, the active focus point of $q_{i}$ (the production level of Firm $i$ ) with conjecturing the production level of Firm $j$ being $q_{j}$, denoted as $c_{i o}\left(q_{i}, q_{j}\right)$ is as follows:

$c_{i o}\left(q_{i}, q_{j}\right)=\arg \max _{c} \min \left[\pi(c), u\left(w_{i}\left(q_{i}, q_{j}, c\right)\right)\right]$.

Likewise, considering Choice 3, the passive focus point of $q_{i}$ (the production level of Firm $i$ ) with conjecturing the production level of Firm $j$ being $q_{j}$, denoted as $c_{i p}\left(q_{i}, q_{j}\right)$ is as follows:

$c_{i p}\left(q_{i}, q_{j}\right)=\arg \min _{c} \max \left[1-\pi(c), u\left(w_{i}\left(q_{i}, q_{j}, c\right)\right)\right]$.

Considering Theorem 1 and (18), the following conclusions can be obtained [9].

(I) Based on the active focus point of $q_{i}$, Firm $i$ chooses its optimal production level $q_{i}^{\Delta}$ to make $u\left(w_{i}\left(q_{i}, q_{j}, c_{i o}\left(q_{i}, q_{j}\right)\right)\right)$ maximize with conjecturing the production level of Firm $j$ being $q_{j}$. That is,

$$
\begin{aligned}
& q_{i}^{\Delta}=\arg \max _{q_{i}} u\left(w_{i}\left(q_{i}, q_{j}, c_{i o}\left(q_{i}, q_{j}\right)\right)\right) \\
& =\frac{\hat{c}_{i o}\left(q_{j}\right)-d q_{j}}{2 d}
\end{aligned}
$$

where $\hat{c}_{i o}\left(q_{j}\right)$, called as active focus point of Firm $i$ with conjecturing the production level of Firm $j$ being $q_{j}$, is the horizontal coordinate of the unique intersection of $\pi(c)$ and $u\left(w_{i}\left(q_{i}^{\nabla}\left(q_{j}, c\right), q_{j}, c\right)\right)$ within $\left[c_{o}, c_{u}\right]$, where $q_{i}^{\nabla}\left(q_{j}, c\right)$ is obtained by (18).

(II) Based on the passive focus point of $q_{i}$, Firm $i$ chooses its optimal production level $q_{i}^{\Delta}$ to make $u\left(w_{i}\left(q_{i}, q_{j}, c_{i p}\left(q_{i}, q_{j}\right)\right)\right)$ maximize with conjecturing the production level of Firm $j$ being $q_{j}$. That is,

$$
\begin{aligned}
& q_{i}^{\Delta}=\arg \max _{q_{i}} u\left(w_{i}\left(q_{i}, q_{j}, c_{i p}\left(q_{i}, q_{j}\right)\right)\right) \\
& =\frac{\hat{c}_{i p}\left(q_{j}\right)-d q_{j}}{2 d}
\end{aligned}
$$

where $\hat{c}_{i p}\left(q_{j}\right)$, called as passive focus point of Firm $i$ with conjecturing the production level of Firm $j$ being $q_{j}$, is the horizontal coordinate of the unique intersection of $1-\pi(c)$ and $u\left(w_{i}\left(q_{i}^{\nabla}\left(q_{j}, c\right), q_{j}, c\right)\right)$ within $\left[c_{l}, c_{o}\right]$, where $q_{i}^{\nabla}\left(q_{j}, c\right)$ is obtained by (18).

In what follows, let us analyze the equilibrium situations of two firms. For the sake of simplification, we assume that the firms have the same type as each other. Suppose that both of firms are normal, it is easy to understand that the Cournot equilibrium for this case, denoted as $\left(q_{1}^{*}, q_{2}^{*}\right)$, is the solution of equations (19) where $c_{o}$ takes the place of $c$. Suppose that both of firms are active or passive, the active and passive Cournot equilibriums, denoted as $\left(q_{1 o}^{*}, q_{2 o}^{*}\right)$ and $\left(q_{1 p}^{*}, q_{2 p}^{*}\right)$, respectively, can be obtained as the following equations:

$$
\begin{gathered}
\left\{\begin{array}{l}
q_{1}=\frac{\hat{c}_{1 o}\left(q_{2}\right)-d q_{2}}{2 d} \\
q_{2}=\frac{\hat{c}_{2 o}\left(q_{1}\right)-d q_{1}}{2 d}
\end{array},\right. \\
\left\{\begin{array}{l}
q_{1}=\frac{\hat{c}_{1 p}\left(q_{2}\right)-d q_{2}}{2 d} \\
q_{2}=\frac{\hat{c}_{2 p}\left(q_{1}\right)-d q_{1}}{2 d}
\end{array},\right.
\end{gathered}
$$

where $\left(q_{1 o}^{*}, q_{2 o}^{*}\right)$ and $\left(q_{1 p}^{*}, q_{2 p}^{*}\right)$ are the solutions of (27) and (28), respectively.

The procedure for solving the equation (27):

Step 1 . Arbitrarily choose a $c \in\left[c_{o}, c_{r}\right]$. 
Step 2. Set $\hat{c}_{i o}(i=1,2)$ as $c$ and solve (27) to obtain $\left(q_{10}^{*}, q_{2 o}^{*}\right)$.

Step 3. Calculate $e(c)=\pi(c)-u\left(w_{i}\left(c, q_{1 o}^{*}, q_{2 o}^{*}\right)\right)$. If $|e(c)| \leq \varepsilon$ then $\hat{c}_{i o}=c$ and stop; if $e(c)>\varepsilon$, then go to Step 4 ; if $e(c)<-\varepsilon$, then go to Step 5 , where $\varepsilon$ is a small positive real number.

Step 4. Set $c$ as $c=c+\Delta c(\Delta c>0)$ and go back to Step 2, where $\Delta c$ is a small positive real number.

Step 5. Set $c$ as $c=c-\Delta c(\Delta c>0)$ and go back to Step 2.

This procedure is explained by Fig.1. It is clear from Fig.1 that $e(c)>0$ implies $c<\hat{c}_{i o}$ and $e(c)<0$ implies $c>\hat{c}_{i o}$.

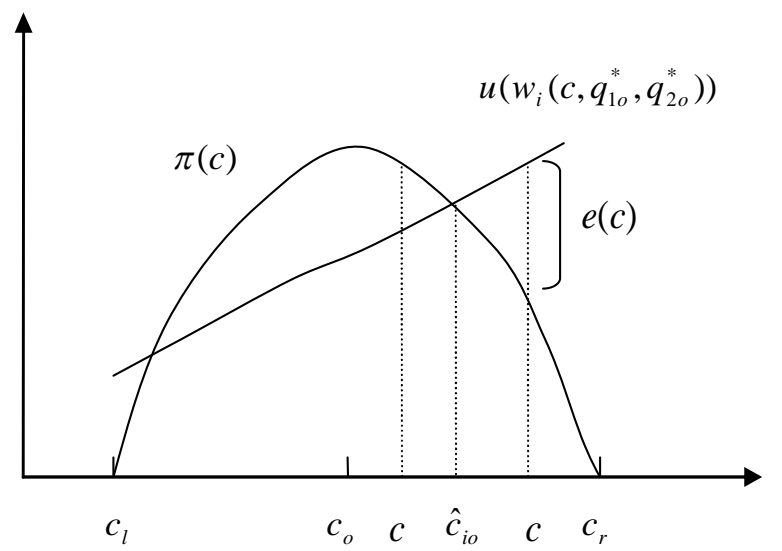

Fig. 1. Graphical explanation of the procedure for obtaining $\hat{c}_{i o}$.

\section{The procedure for solving the equation (28):}

Step 1. Arbitrarily choose a $c \in\left[c_{l}, c_{o}\right]$.

Step 2. Take $\hat{c}_{i p}(\mathrm{i}=1,2)$ as $c$ and solve (28) to obtain $\left(q_{1 p}^{*}, q_{2 p}^{*}\right)$.

Step 3. Calculate $e(c)=1-\pi(c)-u\left(w_{i}\left(c, q_{1 p}^{*}, q_{2 p}^{*}\right)\right)$. If $|e(c)| \leq \varepsilon$ then $\hat{c}_{i p}=c$ and stop; if $e(c)>\varepsilon$, then go to Step 4; if $e(c)<-\varepsilon$, then go to Step 5 .

Step 4. Set $c$ as $c=c+\Delta c(\Delta c>0)$ and go back to Step 2.

Step 5. Set $c$ as $c=c-\Delta c(\Delta c>0)$ and go back to Step 2.

This procedure is explained by Fig.2. It is clear from Fig.2 that $e(c)>0$ implies $c<\hat{c}_{i p}$ and $e(c)<0$ implies $c>\hat{c}_{i p}$.

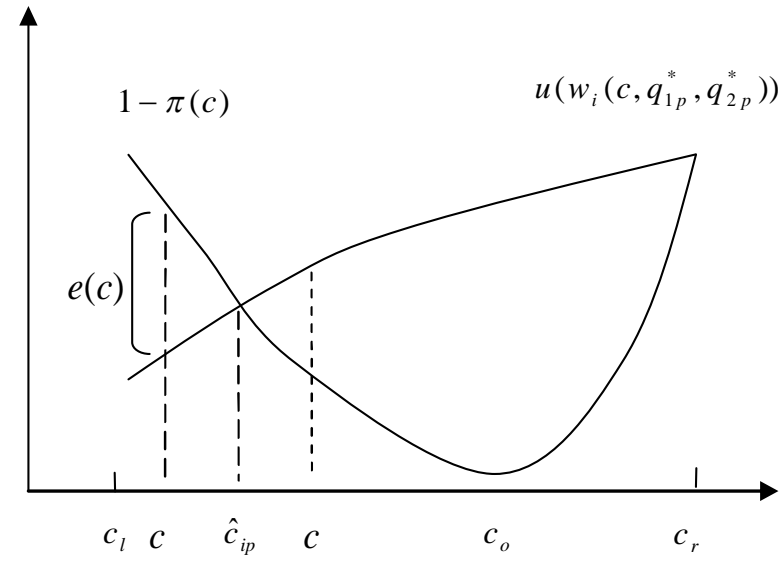

Fig. 2. Graphical explanation of the procedure for obtaining $\hat{c}_{i p}$.

\subsection{Cournot Equilibrium with Asymmetrical Possibilistic Information}

Let us consider an asymmetrical information case. That is, some new information $s$ on $c$ is known by Firm 2 but not by Firm 1. In this case, the production level of Firm 2 is a function of $s$, that is, $q_{2}=q_{2}(s)$. The profit of Firms 1 and 2 are as follows:

$$
\begin{aligned}
& w_{1}\left(q_{1}, q_{2}, c\right)=c q_{1}-d q_{1}^{2}-d q_{1} q_{2}(s), \\
& w_{2}\left(q_{1}, q_{2}, c\right)=c q_{2}(s)-d q_{2}^{2}(s)-d q_{1} q_{2}(s) .
\end{aligned}
$$

For Firm 1, the certain value of $s$ is unknown but its possibility distribution $\pi_{S}(s)$ is known. The possibility distribution of the production level of Firm 2, denoted as $\pi_{Q_{2}}(q)$, can be calculated by the extension principle $\pi_{Q_{2}}(q)=\max _{s . t . q=q_{2}(s)}\left(\pi_{s}(s)\right)$.

Definition 4. Conjecturing the production level of Firm 2 being $q_{2}$ and considering the possibility degree of $q_{2}$, the active focus point of the production level $q_{1}$ with information $s$, denoted as $c_{1 o}^{s}\left(q_{1}, q_{2}\right)$ is defined as follows:

$$
\begin{aligned}
& c_{1 o}^{s}\left(q_{1}, q_{2}\right) \\
& =\arg \max _{c} \min \left[\pi(c), \pi_{Q_{2}}\left(q_{2}\right) \cdot u\left(w_{1}\left(q_{1}, q_{2}, c\right)\right)\right]^{\prime}
\end{aligned}
$$

where $\pi_{Q_{2}}\left(q_{2}\right)$ is regarded as a discount coefficient of the satisfaction level of Firm 1. 
Based on the active focus points $c_{1 o}^{s}\left(q_{1}, q_{2}\right)$, Firm 1 chooses its optimal production level $q_{1}^{\Delta}$ to make $u\left(w_{1}\left(q_{1}, q_{2}, c_{1 o}^{s}\left(q_{1}, q_{2}\right)\right)\right)$ maximize. That is,

$q_{1}^{\Delta}=\arg \max _{q_{1}} u\left(w_{1}\left(q_{1}, q_{2}, c_{1 o}^{s}\left(q_{1}, q_{2}\right)\right)\right)=h_{1 o}\left(q_{2}\right)$

Definition 5. Conjecturing the production level of Firm 2 being $q_{2}$ and considering the possibility degree of $q_{2}$, the passive focus point of the production level $q_{1}$ with information $s$, denoted as $c_{1 p}^{s}\left(q_{1}, q_{2}\right)$ is defined as follows:

$c_{1 p}^{s}\left(q_{1}, q_{2}\right)$

$=\arg \min \max \left[1-\pi(c), \pi_{Q_{2}}\left(q_{2}\right) \cdot u\left(w_{1}\left(q_{1}, q_{2}, c\right)\right)\right]$

Based on the passive focus points $c_{1 p}^{s}\left(q_{1}, q_{2}\right)$, Firm 1 choose its optimal production level $q_{1}^{\Delta}$ to make $u\left(w_{1}\left(q_{1}, q_{2}, c_{1 p}^{s}\left(q_{1}, q_{2}\right)\right)\right)$ maximize. That is,

$q_{1}^{\Delta}=\arg \max _{q_{1}} u\left(w_{1}\left(q_{1}, q_{2}, c_{1 p}^{s}\left(q_{1}, q_{2}\right)\right)\right)=h_{1 p}\left(q_{2}\right)$.

Firm 2 can refine $\pi(c)$ by using the additional information $s$. The renewed information is a kind of conditional possibility distribution, denoted as $\pi_{C \mid s}(c)$, which means that if information is $s$, then the possibility distribution of $c$ is $\pi_{C \mid s}(c)$.

\section{Definition 6.}

Conjecturing the production level of Firm 1 being $q_{1}$, based on the conditional possibility distribution $\pi_{C \mid s}(c)$, the active focus point of the production level of Firm 2, denoted as $c_{2 o}^{s}\left(q_{1}, q_{2}\right)$, is defined as follows:

$$
\begin{aligned}
& c_{2 o}^{s}\left(q_{1}, q_{2}\right) \\
& =\arg \max _{c} \min \left[\pi_{C \mid s}(c), u\left(w_{2}\left(q_{1}, q_{2}, c\right)\right)\right]
\end{aligned}
$$

Based on the active focus points $c_{2 o}^{s}\left(q_{1}, q_{2}\right)$, Firm 2 choose its optimal production level $q_{2}^{\Delta}$ to make $u\left(w_{2}\left(q_{1}, q_{2}, c_{2 o}^{s}\left(q_{1}, q_{2}\right)\right)\right)$ maximize. That is, $q_{2}^{\Delta}=\arg \max _{q_{2}} u\left(w_{2}\left(q_{1}, q_{2}, c_{2 o}^{s}\left(q_{1}, q_{2}\right)\right)\right)=k_{2 o}\left(q_{1}\right)$.

\section{Definition 7.}

Conjecturing the production level of Firm 1 being $q_{1}$, based on the conditional possibility distribution $\pi_{C \mid s}(c)$, the passive focus point of the production level of Firm 2, denoted as $c_{2 p}^{s}\left(q_{1}, q_{2}\right)$, is defined as follows:

$$
\begin{aligned}
& c_{2 p}^{s}\left(q_{1}, q_{2}\right) \\
& =\arg \min _{c} \max \left[1-\pi_{C \mid s}(c), u\left(w_{2}\left(q_{1}, q_{2}, c\right)\right)\right]
\end{aligned}
$$

Based on the passive focus points $c_{2 p}^{s}\left(q_{1}, q_{2}\right)$, Firm 2 choose its optimal production level $q_{2}^{\Delta}$ to make $u\left(w_{2}\left(q_{1}, q_{2}, c_{2 p}^{s}\left(q_{1}, q_{2}\right)\right)\right)$ maximize. That is,

$$
q_{2}^{\Delta}=\arg \max _{q_{2}} u\left(w_{2}\left(q_{1}, q_{2}, c_{2 p}^{s}\left(q_{1}, q_{2}\right)\right)\right)=k_{2 p}\left(q_{1}\right) .
$$

Definition 8. The solutions of the following equations (39) and (40), denoted as $\left(q_{1 o}^{s^{*}}, q_{2 o}^{s^{*}}\right)$ and $\left(q_{1 p}^{s^{*}}, q_{2 p}^{s^{*}}\right)$, are called as active and passive Cournot equilibriums with information $S$, respectively.

$$
\begin{gathered}
\left\{\begin{array}{l}
q_{1}=h_{1 o}\left(q_{2}\right) \\
q_{2}=k_{2 o}\left(q_{1}\right)
\end{array},\right. \\
\left\{\begin{array}{l}
q_{1}=h_{1 p}\left(q_{2}\right) \\
q_{2}=k_{2 p}\left(q_{1}\right)
\end{array} .\right.
\end{gathered}
$$

Lemma 1. If $\pi_{S}(s)=1$ holds for $\forall s$, then $\pi_{Q}(q)=1$ holds for $\forall q$. In this case, (31) and (33) are equal to (23) and (24), respectively.

As a special case, we define $\pi_{C \mid s}(c)$ as follows:

$$
\pi_{C \mid s}(c)=\left(\begin{array}{cl}
1 & c=c_{o} \\
\alpha \pi_{C}(c) & c \neq c_{o}
\end{array},\right.
$$

where $\alpha \leq 1$ is the information factor. The smaller $\alpha$, the more informed $\pi_{C \mid s}(c)$ is.

Theorem 2. Assume that $\pi_{C \mid s}(c)$ is expressed by (41), we have

(I) based on the active focus point of $q_{1}$ expressed by (31), Firm 1 chooses its optimal production level $q_{1}^{\Delta}$ to make $u\left(w_{1}\left(q_{1}, q_{2}, c_{1 o}^{s}\left(q_{1}, q_{2}\right)\right)\right)$ maximize with 
conjecturing the production level of Firm 2 being $q_{2}$. That is,

$$
\begin{aligned}
& q_{1}^{\Delta}=\arg \max _{q_{1}} u\left(w_{1}\left(q_{1}, q_{2}, c_{1 o}^{s}\left(q_{1}, q_{2}\right)\right)\right) \\
& =\frac{\hat{c}_{1_{o}}^{s}\left(q_{2}\right)-d q_{2}}{2 d}
\end{aligned}
$$

where $\hat{c}_{1 o}^{s}\left(q_{2}\right)$, called as active focus point of Firm 1 with asymmetric information $s$, is the horizontal coordinate of the unique intersection of $\pi(c)$ and $\pi_{Q_{2}}\left(q_{2}\right) \cdot u\left(w_{1}\left(q_{1}^{\nabla}\left(q_{2}, c\right), q_{2}, c\right)\right)$ within $\left[c_{o}, c_{u}\right]$, where $q_{1}^{\nabla}\left(q_{2}, c\right)$ is obtained by (18).

(II) based on the active focus point of $q_{2}$ expressed by (35), Firm 2 chooses its optimal production level $q_{2}^{\Delta}$ to make $u\left(w_{2}\left(q_{1}, q_{2}, c_{2 o}^{s}\left(q_{1}, q_{2}\right)\right)\right)$ maximize with conjecturing the production level of Firm 1 being $q_{1}$. That is,

$$
\begin{aligned}
& q_{2}^{\Delta}=\arg \max _{q_{2}} u\left(w_{2}\left(q_{1}, q_{2}, c_{2 o}^{s}\left(q_{1}, q_{2}\right)\right)\right) \\
& =\frac{\hat{c}_{2 o}^{s}\left(q_{1}\right)-d q_{1}}{2 d}
\end{aligned},
$$

where $\hat{c}_{2 o}\left(q_{1}\right)$, called as active focus point of Firm 2 with asymmetric information $s$, is the horizontal coordinate of the unique intersection of $\pi_{C \mid s}(c)$ expressed by (41) and $u\left(w_{2}\left(q_{1}, q_{2}^{\nabla}\left(q_{1}, c\right), c\right)\right)$ within $\left[c_{o}, c_{u}\right]$, where $q_{2}^{\nabla}\left(q_{1}, c\right)$ is obtained by (18).

(III) based on the passive focus point of $q_{1}$ expressed by (33), Firm 1 chooses its optimal production level $q_{1}^{\Delta}$ to make $u\left(w_{1}\left(q_{1}, q_{2}, c_{1 p}^{s}\left(q_{1}, q_{2}\right)\right)\right)$ maximize with conjecturing the production level of Firm 2 being $q_{2}$. That is,

$$
\begin{aligned}
& q_{1}^{\Delta}=\arg \max _{q_{1}} u\left(w_{1}\left(q_{1}, q_{2}, c_{1 p}^{s}\left(q_{1}, q_{2}\right)\right)\right) \\
& =\frac{\hat{c}_{1 p}^{s}\left(q_{2}\right)-d q_{2}}{2 d}
\end{aligned}
$$

where $\hat{c}_{1 p}^{s}\left(q_{2}\right)$, called as passive focus point of Firm 1 with asymmetric information $s$, is the horizontal coordinate of the unique intersection of $1-\pi(c)$ and
$\pi_{Q_{2}}\left(q_{2}\right) \cdot u\left(w_{1}\left(q_{1}^{\nabla}\left(q_{2}, c\right), q_{2}, c\right)\right)$ within $\left[c_{l}, c_{o}\right]$, where $q_{1}^{\nabla}\left(q_{2}, c\right)$ is obtained by (18).

(IV) based on the passive focus point of $q_{2}$ expressed by (37), Firm 2 chooses its optimal production level $q_{2}^{\Delta}$ to make $u\left(w_{2}\left(q_{1}, q_{2}, c_{2 p}^{s}\left(q_{1}, q_{2}\right)\right)\right)$ maximize with conjecturing the production level of Firm 1 being $q_{1}$. That is,

$$
\begin{aligned}
& q_{2}^{\Delta}=\arg \max _{q_{2}} u\left(w_{2}\left(q_{1}, q_{2}, c_{2 p}^{s}\left(q_{1}, q_{2}\right)\right)\right) \\
& =\frac{\hat{c}_{2 p}^{s}\left(q_{1}\right)-d q_{1}}{2 d}
\end{aligned}
$$

where $\hat{c}_{2 p}^{s}\left(q_{1}\right)$, called as passive focus point of Firm 2 with asymmetric information $s$, is the horizontal coordinate of the unique intersection of $1-\pi_{C \mid s}(c)$ and $u\left(w_{2}\left(q_{1}, q_{2}^{\nabla}\left(q_{1}, c\right), c\right)\right)$ within $\left[c_{l}, c_{o}\right]$, where $q_{2}^{\nabla}\left(q_{1}, c\right)$ is obtained by (18).

Proof. It is trivial to prove (42)-(45) with considering Theorem 1 and Definitions 4-7.

(42)-(45) are used for obtaining Cournot equilibriums with asymmetrical possibilistic information by (39) and (40).

\section{Conclusions}

In this paper, within the one-shot framework possibilistic decision models are proposed for analyzing a duopoly market of a new product with a short life cycle. The uncertainty of market is characterized by the possibility distribution of the parameter in the demand function. Three focus points, called normal, active and passive focus points are introduced to show which values should be considered for making a decision with possibilistic information. Based on three kinds of focus points, possibilistic Cournot equilibriums are proposed to analyze the optimal production level of each firm in a duopoly market with asymmetrical possibilistic information. It can be seen that one-shot decision approaches are useful for the situations where a decision is experienced only once and the probability distribution is unavailable due to lack of enough information. 


\section{References}

1. M. Allais, Le comportement de l'homme rationnel devant le risque: Critique des postulats et axiomes de l'école américaine, Econometrica 21 (1953) 503-546.

2. D. Bell, Regret in decision making under uncertainty, Operations Research 30 (1982) 961-981.

3. D. Dubois and H. Prade, Possibility Theory (Plenum Press, New York, 1988).

4. D. Dubois, H. Prade and R. Sabbadin, Decision-theoretic foundations of possibilty theory, European Journal of Operational Research 128 (2001) 459-478.

5. D. Ellsberg, Risk, ambiguity and Savage axioms, Quarterly Journal of Economics 75 (1961) 643-669.

6. I. Gilboa, Expected utility with purely subjective nonadditive probabilities, Journal of Mathematical Economics 16 (1987) 65-88.

7. L. Godo and A. Zapico, On the possibilistic-based decision model: characterization of preference relations under partial inconsistency, Applied Intelligence $\mathbf{1 4}$ (2001) 319-333.

8. T. Gürbüz, Multiple criteria human performance evaluation using Choquet integral, International Journal of Computational Intelligence Systems 3 (2010) 290-300.

9. P. Guo, One-shot decision approach and its application to duopoly market, International Journal of Information and Decision Sciences 2 (2010) 213-232.

10. P. Guo, Private real estate investment analysis within one-shot decision framework, International Real Estate Review 13 (2010) 238-260.

11. P. Guo, One-shot decision theory, IEEE Transactions on Systems, Man and Cybernetics: Part A (In Press)

12. P. Guo, H. Tanaka and H. -J. Zimmermann, Upper and lower possibility distributions of fuzzy decision variables in upper level decision problems, Fuzzy Sets and Systems 111 (2000) 71-79.

13. P. Guo, D. Zeng and H. Shishido, Group decision with inconsistent knowledge, IEEE Transactions on Systems, Man and Cybernetics, Part A: Systems and Humans 32 (2002) 670-679.

14. P. Guo and H. Tanaka, Decision analysis based on fused double exponential possibility distributions, European Journal of Operational Research 148 (2003) 467-479.

15. B. E. Kaha and R. K. Sarin, Modelling ambiguity in decision under uncertainty, Journal of Consumer Research 17 (1988) 265-272.

16. D. Kahneman and A. Tversky, Prospect Theory: An analysis of decision under risk, Econometrica 47 (1979) 263-291.

17. T. Kaya, Multi-attribute evaluation of website quality in E-business using an integrated fuzzy AHPTOPSIS methodology, International Journal of Computational Intelligence Systems 3 (2010) 301-314.

18. X. Li, D. Ruan, J. Liu and Y. Xu, A linguistic-valued weighted aggregation operator to multiple attribute group decision making with quantitative and qualitative information, International Journal of Computational Intelligence Systems 1 (2008) 274 -284.
19. V. Lopez, M. Santos and J. Montero, Fuzzy specification in real estate market decision making, International Journal of Computational Intelligence Systems 3 (2010) 8-20.

20. G. J. Klir, and T. Folger, Fuzzy Sets, Uncertainty and Information (Prentice-Hall, New Jersey, 1988).

21. F. H. Knight, Risk, Uncertainty and Profit (Houghton Mifflin, Boston, 1921).

22. D. A. Kreps, Course in Microeconomic Theory (Princeton University Press, New Jersey, 1990).

23. G. Loomes and R. Sugden, Regret theory: an alternative theory of rational choice under uncertainty, Economical Journal 92 (1982) 805-824.

24. A. Pahlavani, A new fuzzy MADM approach and its application to project selection problem, International Journal of Computational Intelligence Systems 3 (2010) 103-114.

25. D. Schmeidler, Subjective probability and expected utility without additivity, Econometrica 57 (1989) 571587.

26. U. Segal, The Ellsberg paradox and risk aversion: an anticipated utility approach, Internal Economic Review 28 (1987) 175-202.

27. H. Tanaka and P. Guo, Possibilistic Data Analysis for Operations Research (Heidelberg; New York, PhysicaVerlag, 1999).

28. T. Whalen, Decision making under uncertainty with various assumptions about available information, IEEE Transaction on Systems, Man and Cybernetics 14 (1984) 888-900.

29. R. R. Yager, Possibilistic decision making, IEEE Transaction on Systems, Man and Cybernetics 9 (1979) 388-392.

30. L. A Zadeh, Fuzzy sets as a basis for a theory of possibility, Fuzzy Sets and Systems 1 (1978) 3-28.

\section{Appendix: The proof of Theorem 1.}

First, let us show

$$
\begin{aligned}
& \max _{a \in A} \max _{x \in S} \min (\pi(x), u(x, a)) \\
& =\max _{x \in S} \min \left(\pi(x), \max _{a \in A} u(x, a)\right)
\end{aligned} .
$$

Set $h(x, a)=\min (\pi(x), u(x, a))$. Considering the following two equations

$$
\begin{aligned}
& \max _{a \in A} \max _{x \in S} h(x, a)=\max _{x \in S} \max _{a \in A} h(x, a), \\
& \max _{x \in S} \max _{a \in A} \min (\pi(x), u(x, a)) \\
& =\max _{x \in S} \min \left(\pi(x), \max _{a \in A} u(x, a)\right)
\end{aligned}
$$

we have (A.1). Then we show 


$$
\begin{aligned}
& \min _{x \in S} \max (1-\pi(x), u(x, a)) \\
& \leq \min _{x \in S} \max \left(1-\pi(x), \max _{a \in A} u(x, a)\right) .
\end{aligned}
$$

Because the relation $u(x, a) \leq \max _{a \in A} u(x, a)$ holds, with considering three cases (1) $1-\pi(x)<u(x, a)$; (2) $1-\pi(x)=u(x, a)$; (3) $1-\pi(x)>u(x, a)$, we have

$$
\begin{aligned}
& \max (1-\pi(x), u(x, a)) \\
& \leq \max \left(1-\pi(x), \max _{a \in A} u(x, a)\right) \\
& (\forall a \in A \text { and } \forall x \in S) .
\end{aligned}
$$

With considering the fact that if $f(x) \leq g(x)$ then $\min f(x) \leq \min g(x)$, it is easy to understand (A.4).

$1-\pi(x)$ is a continuous, decreasing, function within $\left[x_{l}, x_{c}\right]$ and $\max _{a \in A} u(x, a)$ is a continuous, strictly increasing, function. The following inequalities

$$
\begin{aligned}
& \max _{a \in A} u\left(x_{l}, a\right)<1-\pi\left(x_{l}\right)=1, \\
& 0=1-\pi\left(x_{c}\right)<\max _{a \in A} u\left(x_{c}, a\right),
\end{aligned}
$$

show that there is one and only one intersection of $1-\pi(x)$ and $\max _{a \in A} u(x, a)$ within $\left[x_{l}, x_{c}\right]$. Denote the horizontal coordinate of this intersection as $x_{\Delta}$, then

$$
1-\pi\left(x_{\Delta}\right)=\max _{a \in A} u\left(x_{\Delta}, a\right) .
$$

$1-\pi(x)$ is a decreasing function within $\left[x_{l}, x_{\Delta}\right]$, which means for all $x \in\left[x_{l}, x_{\Delta}\right]$

$$
\begin{aligned}
& \max \left(1-\pi(x), \max _{a \in A} u(x, a)\right) \geq 1-\pi\left(x_{\Delta}\right) . \\
& \max _{a \in A} u(x, a) \text { is an increasing function within } \\
& x \in\left[x_{\Delta}, x_{u}\right], \text { which means for all } x \in\left[x_{\Delta}, x_{u}\right], \\
& \max \left(1-\pi(x), \max _{a \in A} u(x, a)\right) \geq \max _{a \in A} u\left(x_{\Delta}, a\right) . \text { (A.10) }
\end{aligned}
$$

(A.8) makes the following hold.

$$
\begin{aligned}
& \min _{x \in S} \max \left(1-\pi(x), \max _{a \in A} u(x, a)\right) \\
& =1-\pi\left(x_{\Delta}\right)=\max _{a \in A} u\left(x_{\Delta}, a\right)
\end{aligned}
$$

which is the second and third equalities of (13). Set $a_{\Delta}=\arg \max _{a \in A} u\left(x_{\Delta}, a\right)$. It can be understood that $x_{\Delta}$ is also the horizontal coordinate of the intersection of $1-\pi(x)$ and $u\left(x, a_{\Delta}\right)$. Similarly, Because $u\left(x, a_{\Delta}\right)$ is a continuous, strictly increasing, function of $x, x_{\Delta}$ satisfies $\min _{x \in S} \max \left(1-\pi(x), u\left(x, a_{\Delta}\right)\right)$, which means if we take $x=x_{\Delta}$ and $a=a_{\Delta}$ then (A.4) becomes equality. Thus, the first equality of (13) can be obtained. It is also easy to understand that an unique intersection of $\pi(x)$ and $\max _{a \in A} u(x, a)$ exists within $\left[x_{c}, x_{u}\right]$. Denote the horizontal coordinate of this intersection as $x^{\Delta}$, it is obvious that (14) holds considering (A.1). It proves Theorem 1. 